\title{
Cost Optimisation for Underground Mining Networks*
}

\author{
M. Brazil, D.A. Thomas, J.F. Weng ${ }^{1}$ \\ ARC Special Research Center for Ultra-Broadband Information Networks, \\ Department of Electrical and Electronic Engineering, The University of Melbourne, \\ Victoria 3010, Australia
}

J.H. Rubinstein

Department of Mathematics and Statistics, The University of Melbourne, Victoria 3010, Australia

D.H. Lee

Department of Mathematics, University of South Australia, SA 5095, Australia

September 26, 2003

\begin{abstract}
In this paper we consider the problem of optimising the construction and haulage costs of underground mining networks. We focus on a model of underground mine networks consisting of ramps in which each ramp has a bounded maximum gradient. The cost depends on the lengths of the ramps, the tonnages hauled through them and their gradients. We model such an underground mine network as an edgeweighted network and show that the problem of optimising the cost of the network can be described as an unconstrained non-linear optimisation problem. We show that, under a mild condition which is satisfied in practice, the cost function is convex. Finally we briefly discuss how the model can be generalised to those underground mine networks that are composed not only of ramps but also vertical shafts, and show that the total cost in the generalised model is still convex under the same condition. The convexity of the cost function ensures that any local minimum is a global minimum for the given network topology, and theoretically any descent algorithms for finding local minima can be applied to the design of minimum cost mining networks.
\end{abstract}

Keywords: convexity, network optimization, underground mining

\section{Introduction}

In underground mines ore-zones are accessed by a network of ramps. Ramps are tunnels used to provide both access to the ore zone and haulage of ore from the ore zone via a fleet of trucks. Clearly, ramps cannot be very steep because of the truck capability and thus are constrained to a maximum absolute gradient (i.e. slope) $m$, which is typically between $1 / 9$ and $1 / 7$ depending on the particular trucks to be used. In many mines, the ore and waste is hauled back up through this ramp network to the surface, however, in large or deep mines one or

1 Corresponding author. Email:weng@ee.mu.oz.au

* This work was supported by the Australian Research Council.

(C) 2003 Kluwer Academic Publishers. Printed in the Netherlands. 


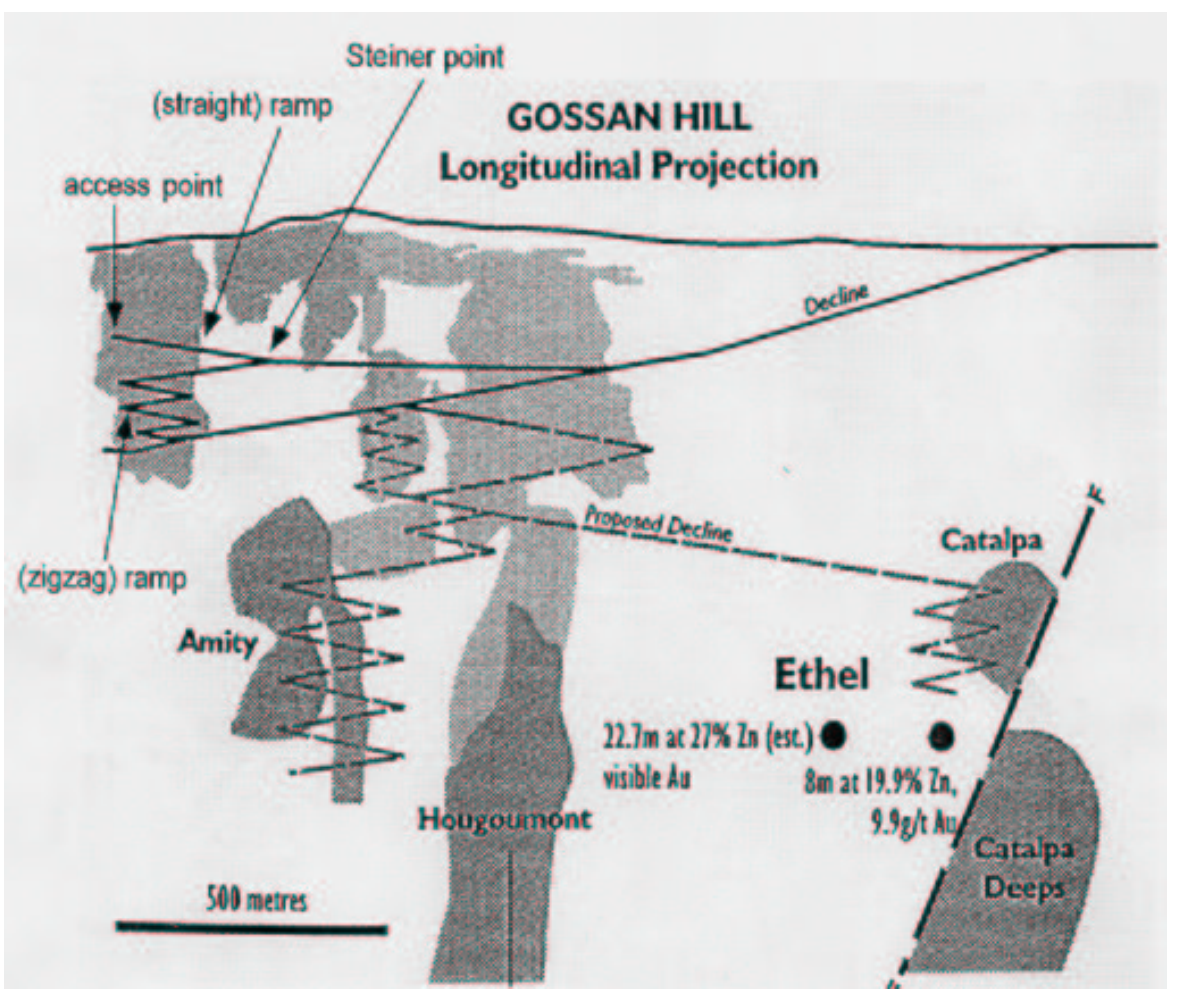

Figure 1. An underground ramp network.

more vertical shafts may be constructed to haul this material. In this paper we concentrate on mines using only ramps for both access and haulage but, in the last section, we indicate how the ideas developed can be generalized to mines with shafts. Figure 1 shows a section view of an actual underground mine with the network of ramps shown by solid lines for the existing network and dashed lines for the proposed development (Normandy, 2001).

A network that models the underground mining infrastructure (the system of ramps/shafts) must be connected - there must be a path from each working level of the mine to the surface. It must also be ventilated and navigable. Navigability requires consideration of curvature - trucks have a minimum turning circle. At the macro design level, which is the focus of this paper, we concentrate on the ramp network design subject to a gradient constraint and assume the designs can be ventilated and made navigable with only minor impact on the optimality of the original design. 
The aim of this work is to find the least cost ramp network to mine out a given set of ore zones. Thus we assume there is a given surface portal and a given set of coordinates at various levels underground indicating the access points to the ore-zones from which the mined material is hauled to the surface through the network. A tonnage is given for each level access point. We seek to minimize a cost comprising both the development cost of building (digging and fitting out) the ramps and the haulage cost of transporting material from each level to the surface over the life of the mine. Development cost is given as a development rate, in $\$$ per metre, multiplied by the ramp length in metres. Haulage cost is modelled as tonnes $\times($ path length $) \times($ haulage rate) where the haulage rate is expressed in $\$$ per tonne.metre units and path length is the sum of the lengths of the ramps through which the material is hauled. A special feature of the minimum cost design which follows is that it admits haulage rates as a (possibly) non-linear function of the gradient of the ramps. Mine designers do not always make this distinction explicit but haulage costs do increase with gradient; steeper grades increase costs with respect to maintenance, tyre wear and fuel use of trucks.

Early in 1989, Lee (Lee, 1989) raised the topic of modelling and optimising the design of underground mining networks. This is an important topic as mining is an economically significant industry worldwide. Our previous work in this area has appeared in (Brazil et al., 1998; Brazil et al., 2000-1; Brazil et al., 2000-2; Brazil et al., 2002). The mathematical model of a mining network in which the gradient constraint is the only constraint on the ramps is referred to as a gradient-constrained network. As in the literature of minimum networks, (Hwang et al., 1992) for example, in our network model the links corresponding to ramps are referred to as edges, and the nodes corresponding to the access points, i.e. the points on the boundaries of the ore deposits from which ore is to be extracted, are fixed points and are referred to as terminals. To minimise the cost, the network is permitted to contain extra variable nodes. These nodes, corresponding to junctions where three or more ramps meet, are referred to as Steiner points. The locations of the Steiner points and the shapes of the curves representing the edges are the variables in the problem of minimising the costs of mining networks. Moreover, the graph structure of a network, which describes how the terminals and Steiner points are interconnected, i.e., the pattern of connections between nodes, is referred to as the topology of the network. In this paper we focus on finding the least cost mining network with respect to a given set of access points and a given topology of the network under the gradient constraint. Global 
optimality for a given set of access points can be achieved by finding the best topology, using, for example, branch-and-bound techniques.

In the first of our previous papers in this area (Brazil et al., 1998) a special case of gradient-constrained ramp networks was studied in which all terminals lie in a vertical plane. In this special case the minimum networks have a strongly restricted canonical form, which allows them to be constructed in linear time if the topology is known. However, even for this special case, the problem of finding a global minimum for the network is NP-complete. In (Brazil et al., 2000-1), we studied the fundamental properties of gradient-constrained ramp networks in 3-space where the cost of each edge is proportional only to its length. It was shown that there are strong geometric restrictions on the possible arrangement of edges around a Steiner point in such a minimum network. The main purpose of the paper (Brazil et al., 2000-2) was to demonstrate the usefulness of software developed by the authors and based on this theory. The paper looks at its application to several real-life case studies of mining networks. In (Brazil et al., 2002) a mathematical model of mining networks was established in which networks contain shafts as well as ramps and the haulage cost on an edge is linear in the gradient of the edge. For this linear model, the convexity of the network cost was proved.

Now in this present paper we first generalise the model of ramp networks to one in which the haulage cost is not limited to being linear but is a general increasing function of the edge gradients. Therefore, a ramp network is regarded as a network whose edge weights are variable, and the least cost mining network problem can be described as a nonlinear optimisation problem with a non-linear constraint. We show that the (gradient) constraint can be removed so that the problem becomes a non-linear unconstrained optimisation problem and we prove that for any given topology the cost function is convex under a mild condition which is satisfied in all known practical applications. In the last section we briefly discuss how the ramp network model can be generalised to those underground mine networks that are composed not only of ramps but also vertical shafts, and show that the total cost in the generalised model is still convex under the same condition. The convexity of the cost function ensures that any local minimum is a global minimum for the given network topology, and theoretically any descent algorithms for finding local minima can be applied to the design of minimum cost mining networks. (The authors, together with N.C.Wormald, have developed such algorithms for ramp networks.) 


\section{Modelling Underground Ramp Networks}

In this section we give a precise description of the mathematical model of an underground ramp network, and express the cost optimisation problem in terms of the properties of this model. Let $T$ be a ramp network in Euclidean 3-space with maximum gradient constraint $m$. $T$ is a network composed of a set of vertices $V$ and a set of edges $E$, each of which interconnects a pair of vertices. $V$ contains two types of vertices: terminals whose locations are fixed in the model, and Steiner points whose locations are free to vary. The aim is to minimise a cost function for the network with respect to the locations of the Steiner points and the shapes of the edges. In the model, we place the following conditions on the edges in $T$ :

- every edge $e_{i}(i=1,2, \ldots)$ in $E$ is a monotone increasing (or decreasing) and piecewise differentiable curve; and

- the absolute gradient $g(p)$ at each differentiable point $p$ on the curve representing $e_{i}$ is no more than the fixed constant $m$, and we assume $m<1$, a condition easily satisfied in all practical applications.

For each edge $e_{i}$ in $T$, define $L\left(e_{i}\right)$ to be the Euclidean length of the curve $e_{i}$, and let $L(T)=\sum_{i} L\left(e_{i}\right)$ be the total length of the ramp network $T$.

The principal life-of-mine costs of an underground mine can be divided up as follows: development costs, haulage costs, and a collection of other costs which are either independent of the network design or relatively insignificant compared to the development and haulage costs. Ignoring the latter costs, we assume the corresponding cost function $C(T)$ of the ramp network model $T$ consists of two components:

1. A development cost $D_{c}(T)$ that is uniformly proportional to the total length $L(T)=\sum_{i} L\left(e_{i}\right)$ of $T$;

2. A haulage cost $H_{c}(T)=\sum_{i} H_{c}\left(e_{i}\right)$ that, for each edge $e_{i}$, is not only dependent on the length $L\left(e_{i}\right)$ of $e_{i}$ but also on the given tonnage $t\left(e_{i}\right)$ of ore that, it is estimated, will be extracted and transported through $e_{i}$ over the life of the mine. Moreover, the haulage cost for each edge $e_{i}$ is also dependent on the gradient at each point of $e_{i}$. Let $f_{g}\left(e_{i}\right)$ denote the dependence of the haulage cost on the gradient $g(p)$ at each point $p$ of $e_{i}$. As discussed in Section 1, the per-meter haulage cost along a ramp increases with gradient. Hence we will assume $f_{g}\left(e_{i}\right)$ is a function which is monotone increasing (and possibly non-linear) in $g(p)$. Summing up all these considerations, we 
assume that the haulage cost for each edge $e_{i}$ is proportional to $t\left(e_{i}\right) f_{g}\left(e_{i}\right) L\left(e_{i}\right)$.

Let $c$ with any subscript or superscript denote a non-negative constant. In particular, let $c_{d}$ be the proportionality constant related to development and $c_{h}$ be the proportionality constant related to haulage. The total cost $C(T)$ of $T$ now can be expressed as

$$
\begin{aligned}
C(T)=D_{c}(T)+H_{c}(T) & =c_{d} \sum_{i} L\left(e_{i}\right)+c_{h} \sum_{i} t\left(e_{i}\right) f_{g}\left(e_{i}\right) L\left(e_{i}\right) \\
& =\sum_{i}\left(c_{d}+c_{h} t\left(e_{i}\right) f_{g}\left(e_{i}\right)\right) L\left(e_{i}\right) .
\end{aligned}
$$

The topology of $T$ is defined to be its structure as an abstract graph, in terms of edges and vertices. For a fixed topology of $T, C(T)$ is a function of the locations of the Steiner points in $T$ and the shape of each curve $e_{i}$. In analyzing $C(T)$ it will be convenient to use the arc lengths of edges as parameters in the problem. More precisely, we parameterize each edge $e_{i}$ with respect to the arc length of the projection of $e_{i}$ onto the horizontal plane. We now show how to write $C(T)$ in terms of such a parameterization.

The arc length function of $e_{i}$ can be denoted by $s(r)$ where $r$ is the arc length function of the horizontal projection of $e_{i}$. Let $z(r)$ be the vertical coordinate of the point on $e_{i}$ corresponding to $r$. We choose the endpoint of $e_{i}$ from which we measure arc length so that $z(r)$ is a non-decreasing function of $r$. The gradient $g(r)$ at the point on $e_{i}$ corresponding to $r$ is the derivative of $z(r)$ with respect to $r$, i.e.

$$
g(r)=\frac{d z}{d r}=\dot{z}(r) \geq 0,
$$

and the linear element $d s$ of $e_{i}$ satisfies $d s^{2}=d r^{2}+d z^{2}$, i.e.

$$
d s=\left(\sqrt{1+\dot{z}^{2}(r)}\right) d r .
$$

It follows that

$$
L\left(e_{i}\right)=\int d s=\int_{0}^{r_{i}} \sqrt{1+\dot{z}^{2}(r)} d r=G\left(r_{i}, z_{i}, 0\right),
$$

where $r_{i}$ is the arc length of the horizontal projection of $e_{i}, z_{i}=z$ is the function of $r$ that determines the vertical coordinate of each point on $e_{i}$, and the function $G\left(r_{i}, z_{i}, j\right)$ is defined as

$$
G\left(r_{i}, z_{i}, j\right) \stackrel{\text { def }}{=} \int_{0}^{r_{i}} \dot{z}_{i}^{j}(r) \sqrt{1+\dot{z}_{i}^{2}(r)} d r .
$$


Because the haulage cost along the ramp corresponding to $e_{i}$ increases faster than the increase in gradient, as discussed in Section 1, $f_{g}\left(e_{i}\right)$ can be effectively approximated by a polynomial in $g(r)$ with non-negative coefficients $c_{i}$ :

$$
f_{g}\left(e_{i}\right)=c_{0}+c_{1} g(r)+c_{2} g^{2}(r)+\cdots+c_{k} g^{k}(r)=\sum_{j=0}^{k} c_{j} \dot{z}^{j}(r) .
$$

It follows that

$$
\begin{aligned}
f_{g}\left(e_{i}\right) L\left(e_{i}\right) & =\int\left(\sum_{j=0}^{k} c_{j} \dot{z}^{j}(r)\right) d s=\int_{0}^{r_{i}}\left(\sum_{j=0}^{k} c_{j} \dot{z}^{j}(r)\right) \sqrt{1+\dot{z}^{2}(r)} d r \\
& =\sum_{j=0}^{k} c_{j} G\left(r_{i}, z_{i}, j\right)
\end{aligned}
$$

Substituting (2) and (4) into (1), the total cost of $T$ becomes

$$
\begin{aligned}
C(T) & =\sum_{i}\left(\left(c_{d}+c_{h} t\left(e_{i}\right) c_{0}\right) G\left(r_{i}, z_{i}, 0\right)+\sum_{j=1}^{k} c_{h} t\left(e_{i}\right) c_{j} G\left(r_{i}, z_{i}, j\right)\right) \\
& =\sum_{i} c_{i}^{*}\left(\sum_{j=0}^{k} \rho_{i j} G\left(r_{i}, z_{i}, j\right)\right),
\end{aligned}
$$

where

$$
\begin{gathered}
c_{i}^{*}=c_{d}+c_{h} t\left(e_{i}\right) c_{0}, \\
\rho_{i 0}=1, \quad \text { and } \rho_{i j}=\frac{c_{h} t\left(e_{i}\right) c_{j}}{c_{d}+c_{h} t\left(e_{i}\right) c_{0}} \quad(1 \leq j \leq k) .
\end{gathered}
$$

In summary, the problem of designing a minimum cost underground ramp network $T$ with a given topology is now expressed as a non-linear optimisation problem as follows:

Objective: Minimise $C(T)=\sum_{i} c_{i}^{*}\left(\sum_{j=0}^{k} \rho_{i j} G\left(r_{i}, z_{i}, j\right)\right)$.

Constraints: The gradient at any differentiable point on the edges in $T$ is no more than a prescribed maximal value $m<1$, i.e., on each edge $e_{i}, g(r) \leq m$.

\section{Basic Properties of Minimum Cost Ramp Networks}

In this section we reformulate the above optimisation problem as an unconstrained problem, and then show that the objective function is 
convex (with respect to variables corresponding to the projected length and gradient of each edge). In order to show that the cost of the entire network is convex it suffices to show that the cost of each edge is convex, since a sum of convex functions is itself convex. The consequence of this convexity is that there is a unique local minimum for $C(T)$ for any fixed topology of $T$.

The simplest non-trivial case of the model occurs when $c_{j}=0$ for all $j \geq 0$ (i.e., the cost function only takes development costs into consideration). In this case

$$
C(T)=\sum_{i} c_{d} G\left(r_{i}, z_{i}, 0\right)=c_{d} \sum_{i} L\left(e_{i}\right)=c_{d} L(T) .
$$

To minimise $C(T)$ we need only minimise the total length of $T$. This has been studied in (Brazil et al., 2000-1) and, for this case, two basic properties of $T,(\mathbf{P 1})$ and (P2), have been proved.

(P1) Let $T$ be a minimum cost ramp network. An edge in $T$ is a straight line if the gradient between its two endpoints is no more than $m$. Otherwise it is a monotone increasing (or decreasing) piecewise differentiable curve such that the gradient at each differentiable point is $m$.

REMARK 3.1. Note that in the latter case the edge does not necessarily lie in a plane. For example, the curve could be an arc of a right circular helix with gradient $m$.

A crucial consequence of Property (P1) is that the gradient on each edge in a minimum ramp network is constant. Hence, in our model we can assume, without loss of generality, that each edge $e_{i}$ has an associated absolute gradient $g_{i}$, such that $g(p)=g_{i}$ for each differentiable point $p$ on $e_{i}$. We can consider the quantities $r_{i}$ and $g_{i}$ to be the variables of the cost function $C(T)$.

(P2) $C(T)$ is a convex function with respect to the variables $r_{i}, g_{i}$.

The main aim of this paper is to prove that these two properties also hold in the general case (Theorem 3.2 and Theorem 3.4). First we refer to a known result in variational calculus.

LEMMA 3.1. [Clegg, 1968] The curve $y(x)$ minimising $\int_{a}^{b} F\left(x, y, y^{\prime}\right) d x$ is a straight line segment between $a$ and $b$ if the integrand $F\left(x, y, y^{\prime}\right)$ is a function only of the derivative $y^{\prime}$.

THEOREM 3.2. Property (P1) holds in the general case. 
Proof. Since all $\rho_{i j}$ in (5) are non-negative, $C(T)$ is minimised if and only if each term on the right-hand side of (5) is minimised. That is, for each $e_{i}$ and each $j \geq 0$ we need minimise

$G\left(r_{i}, z_{i}, j\right)=\int_{0}^{r_{i}} F\left(r, z_{i}, \dot{z}_{i}\right) d r, \quad F\left(r, z_{i}, \dot{z}_{i}\right) \stackrel{\text { def }}{=} \dot{z}_{i}^{j}(r) \sqrt{1+\dot{z}_{i}^{2}(r)}, j \geq 0$.

Note that $F\left(r, z_{i}, \dot{z}_{i}\right)$ contains only $\dot{z}_{i}$. Hence, if $\left(z_{i}\left(r_{i}\right)-z_{i}(0)\right) / r_{i} \leq$ $m$, then by Lemma $3.1 z_{i}$ is linear in $r$ and the edge $e_{i}$ is a straight line. If $\left(z_{i}\left(r_{i}\right)-z_{i}(0)\right) / r_{i}>m$, then the edge can be partitioned into a monotone increasing (or decreasing) zigzag line so that for each segment the absolute gradient between its two endpoints is $m$ (see Figure 1) and again, by Lemma 3.1, each segment is a straight line. Since each straight line segment has absolute gradient $m$, the length of each segment is $\sqrt{1+m^{-2}}\left(z_{i}\left(r_{i}\right)-z_{i}(0)\right)$, depending only on the height $\left(z_{i}\left(r_{i}\right)-z_{i}(0)\right)$. It also follows that the zigzag line does not necessarily lie in the plane determined by its two endpoints. As the number of segments approaches infinity and the length of the largest segment approaches zero, $e_{i}$ becomes a piecewise differentiable curve. This proves the theorem.

REMARK 3.2. This theorem is not trivial. For example, if $F\left(r, z_{i}, \dot{z}_{i}\right)$ were dependent also on $z_{i}$, then the edges in $T$ would not be straight for minimising $G\left(r_{i}, z_{i}, j\right)$. The reader can refer to (Clegg, 1968, pp.13-21).

Let $w_{i}$ be the Euclidean endpoint-gradient of $e_{i}$, i.e. if the endpoints of $e_{i}$ have coordinates $\left(p_{1}, p_{2}, p_{3}\right)$ and $\left(q_{1}, q_{2}, q_{3}\right)$, define

$$
w_{i}=\frac{\left|q_{3}-p_{3}\right|}{\sqrt{\left(q_{1}-p_{1}\right)^{2}+\left(q_{2}-p_{2}\right)^{2}}} .
$$

In other words, $w_{i}$ is the absolute gradient of the Euclidean line segment between the endpoints of $e_{i}$.

Let $h_{i}=z_{i}\left(r_{i}\right)-z_{i}(0) \geq 0$ be the difference in height between the endpoints of $e_{i}$. Observe that if $w_{i} \leq m$ then $w_{i}=h_{i} / r_{i}$ since $e_{i}$ is a straight line segment. We also have the following result.

COROLLARY 3.3. At any differentiable point on an edge $e_{i}$, the absolute gradient is:

$$
g_{i}= \begin{cases}w_{i}, & \text { if } w_{i} \leq m \\ m, & \text { if } w_{i} \geq m\end{cases}
$$


By this corollary we can now write the optimisation problem solely in terms of an objective function

$$
C(T)=\sum_{i} c_{i}^{*}\left(\sum_{j=0}^{k} \rho_{i j} g_{i}^{j} L\left(e_{i}\right)\right)
$$

where

$$
L\left(e_{i}\right)= \begin{cases}\sqrt{r_{i}^{2}+h_{i}^{2}}=\left(\sqrt{1+w_{i}^{-2}}\right) h_{i}, & \text { if } w_{i} \leq m ; \\ \left(\sqrt{1+m^{-2}}\right) h_{i}, & \text { if } w_{i} \geq m .\end{cases}
$$

The following theorem shows that Property (P2) holds in the general case

THEOREM 3.4. $C(T)$ is convex if for every edge $e_{i}$

$$
\max _{j}\left\{\rho_{i j}\right\} \leq \frac{(1-m)^{2}}{m^{2}+m^{3}} .
$$

Proof. If $c_{0} \neq 0$ but $c_{j}=0$ for all $j \geq 1$, then

$$
C(T)=\sum_{i} c_{i}^{*} L\left(e_{i}\right)
$$

In this case, it is easily seen that each term $L\left(e_{i}\right)$ is convex and $C(T)$ is convex. In the general case, however, some $c_{j} \neq 0$ and $g_{i}^{j} L\left(e_{i}\right)$ might be non-convex. In that case, we will show that the $\operatorname{sum} \sum_{j=0}^{k} \rho_{i j} g_{i}^{j} L\left(e_{i}\right)$ is still convex under the condition (9). Specifically, for any $i$ let

$$
\begin{aligned}
& F_{1 i}=\sum_{j=0}^{k} \rho_{i j} w_{i}^{j} \sqrt{1+w^{-2}} h_{i}, \\
& F_{2 i}=\sum_{j=0}^{k} \rho_{i j} m^{j} \sqrt{1+m^{-2}} h_{i} .
\end{aligned}
$$

Below in Lemma 3.5 and Lemma 3.6 we prove that $F_{1 i}$ is convex when $w_{i} \leq m$ and $F_{2 i}$ is convex when $w_{i} \geq m$. Moreover, in Lemma 3.7 we show that under condition (9):

$$
C(T)=\sum_{i} c_{i}^{*}\left(\sum_{j=0}^{k} \rho_{i j} g_{i}^{j} L\left(e_{i}\right)\right)=\sum_{i} c_{i}^{*} \max \left\{F_{1 i}, F_{2 i}\right\} .
$$

Therefore, the theorem is true because the maximum of two convex functions is convex, ensuring convexity for the cost of each edge, and the sum of convex functions is convex, which implies convexity for the cost function on the whole network $T$. 
LEMMA 3.5. For any $i F_{1 i}$ is convex under the condition (9) when $w_{i} \leq m$.

Proof. For simplicity, we omit the subscript $i$ in the proof. Suppose $e=P Q$ is an edge in $T$ satisfying $w \leq m<1$. After transforming and re-scaling the coordinates we may assume that one endpoint $P$ is the origin $(0,0,0)$ and the other endpoint $Q$ is $(1,0, w)$. Then $r=1$ and $L=\sqrt{1+w^{2}}$. Let $Q$ be perturbed in direction

$$
\mathbf{v}=\left(\cos \phi_{x}, \cos \phi_{y}, \cos \phi_{z}\right)=(\alpha, \beta, \gamma),
$$

where $\phi_{x}, \phi_{y}, \phi_{z}$ are the angles of $\mathbf{v}$ with the $\mathrm{x}-, \mathrm{y}-, \mathrm{z}$-axis respectively. Then as the perturbed point, the coordinates of $Q$ are $(1+t \alpha, t \beta, w+$ $t \gamma)$, and

$$
\begin{aligned}
h(t) & =w+t \gamma, \\
r(t) & =\sqrt{(1+t \alpha)^{2}+(t \beta)^{2}}, \\
L(t) & =\sqrt{r^{2}(t)+(w+t \gamma)^{2}},
\end{aligned}
$$

where $t=|\mathbf{v}|$. Define

$$
f(j, t) \stackrel{\text { def }}{=} \frac{h^{j}(t)}{r^{j}(t)} L(t) .
$$

Then, what we need to prove is that the second directional derivative of $F_{1}(t)$

$$
\frac{d^{2}}{d t^{2}} F_{1}(t)=\sum_{j=0}^{k} \rho_{j} \frac{d^{2}}{d t^{2}} f(j, t)
$$

is non-negative under the condition (9). It is easy to derive that with respect to $t$,

$$
\begin{gathered}
\left.\frac{d}{d t} r(t)\right|_{t=0}=\alpha, \\
\left.\frac{d^{2}}{d t^{2}} r(t)\right|_{t=0}=\beta^{2}, \\
\left.\frac{d}{d t} L(t)\right|_{t=0}=\frac{\alpha+w \gamma}{L}, \\
\left.\frac{d^{2}}{d t^{2}} L(t)\right|_{t=0}=\frac{(\gamma-w \alpha)^{2}+\beta^{2} L^{2}}{L^{3}} .
\end{gathered}
$$

Because

$$
\frac{d}{d t} f(j, t)=\left(\frac{j h^{j-1} \gamma}{r^{j}}-\frac{j h^{j}}{r^{j+1}} \frac{d r}{d t}\right) L+\frac{h^{j}}{r^{j}} \frac{d L}{d t},
$$




$$
\begin{aligned}
\frac{d^{2}}{d t^{2}} f(j, t) & =\frac{(j-1) j \gamma^{2} h^{j-2} L}{r^{j}} \\
& +\frac{2 j h^{j-1}}{r^{j+1}}\left(\gamma r \frac{d L}{d t}-\gamma L \frac{d r}{d t}-h \frac{d r}{d t} \frac{d L}{d t}\right) \\
& -\frac{j h^{j}}{r^{j+1}} \frac{d^{2} r}{d t^{2}}+\frac{h^{j}}{r^{j}} \frac{d^{2} L}{d t^{2}},
\end{aligned}
$$

when $t=0$, after simplification we have

$$
\begin{aligned}
L^{3}\left(\frac{d^{2}}{d t^{2}} f(j, 0)\right)= & w^{j-2}(\gamma-\alpha w)^{2}\left(j(j-1)+j w^{4}+j^{2}\left(2 w^{2}+w^{4}\right)+w^{2}\right) \\
& +\beta^{2} w^{j}\left(1-j-j w^{2}\right)\left(1+w^{2}\right) \\
\geq & \beta^{2} w^{j}\left(1-j-j w^{2}\right)\left(1+w^{2}\right)
\end{aligned}
$$

because $w \leq m<1$ implies that $j(j-1)+j w^{4}+j^{2}\left(2 w^{2}+w^{4}\right)+w^{2} \geq 0$ and the first term is non-negative. By re-scaling if necessary we can assume that $\rho_{0}=1$. Note also that

$$
w^{j}\left(1-j-j w^{2}\right) \begin{cases}=1, & \text { if } j=0 \\ <0, & \text { if } j \geq 1 .\end{cases}
$$

Let $\rho=\max _{j}\left\{\rho_{j}\right\}$. Then,

$$
\begin{aligned}
\frac{d^{2}}{d t^{2}} & F_{1}(t) \\
& =\sum_{j=0}^{k} \rho_{j} \frac{d^{2}}{d t^{2}} f(j, 0) \\
& \geq \frac{\beta^{2}\left(1+w^{2}\right)}{L^{3}} \sum_{j=0}^{k} \rho_{j} w^{j}\left(1-j-j w^{2}\right) \\
& \geq \frac{\beta^{2}\left(1+w^{2}\right)}{L^{3}}\left(1+\rho\left(\sum_{j=1}^{k} w^{j}-\left(1+w^{2}\right) \sum_{j=1}^{k} j w^{j}\right)\right) \quad\left(\text { since } \rho \geq \rho_{j}\right) \\
& =\frac{\beta^{2}\left(1+w^{2}\right)}{L^{3}}\left(1+\rho\left(\frac{w}{1-w}-\frac{w^{k+1}}{1-w}-\frac{\left(1+w^{2}\right) w}{(1-w)^{2}}\right.\right. \\
& =\frac{\beta^{2}\left(1+w^{2}\right)}{L^{3}}\left(1+\rho\left(\frac{w}{1-w}-\frac{w\left(1+w^{2}\right)}{(1-w)^{2}}\right)\right. \\
& \left.+\frac{\rho w^{k+1}\left(w+w^{2}+k(1-w)\left(1+w^{2}\right)\right)}{(1-w)^{2}}\right)
\end{aligned}
$$




$$
\begin{aligned}
& \geq \frac{\beta^{2}\left(1+w^{2}\right)}{L^{3}}\left(1+\rho\left(\frac{w}{1-w}-\frac{w\left(1+w^{2}\right)}{(1-w)^{2}}\right)\right) \quad(\text { since } w \leq m<1) \\
& =\frac{\beta^{2}\left(1+w^{2}\right)}{L^{3}}\left(1-\frac{\rho(1+w) w^{2}}{(1-w)^{2}}\right) \\
& \geq 0
\end{aligned}
$$

if $\rho \leq(1-w)^{2} /\left(w^{2}+w^{3}\right)$. Hence the lemma holds since $w \leq m<1$ and

$(1-w)^{2} /\left(w^{2}+w^{3}\right) \geq(1-m)^{2} /\left(m^{2}+m^{3}\right)$.

LEMMA 3.6. For each $i F_{2 i}$ is convex when $w_{i} \geq m$.

Proof. Since each term in $F_{2 i}$ is linear in $h_{i}$ and $h_{i}(t)$ is linear in $t$ by (10), it follows that the first directional derivative of $F_{2 i}$ is constant and the second directional derivative of $F_{2 i}$ is zero.

LEMMA 3.7. $C(T)=\sum_{i} c_{i}^{*} \max \left\{F_{1 i}, F_{2 i}\right\}$ under the condition (9).

Proof. As in Lemma 3.5 we omit the subscript $i$. By the definitions of $F_{1}, F_{2}$

$$
F_{1}-F_{2}=h \sum_{j=0}^{k} \rho_{j}\left(w^{j} \sqrt{1+w^{-2}}-m^{j} \sqrt{1+m^{-2}}\right) .
$$

What we need to prove is that

$$
F \stackrel{\text { def }}{=} \frac{F_{1}-F_{2}}{h} \begin{cases}\geq 0, & \text { if } w \leq m \\ \leq 0, & \text { if } w \geq m\end{cases}
$$

Note that

$$
\frac{m^{k+1} \sqrt{1+m^{-2}}}{1-m}-\frac{w^{k+1} \sqrt{1+w^{-2}}}{1-w} \begin{cases}\geq 0, & \text { if } w \leq m \\ \leq 0, & \text { if } w \geq m\end{cases}
$$

Clearly, $F$ is symmetric with respect to $w$ and $m$, hence we need only to prove the case of $w \leq m<1$. It is easy to check that

$$
w^{j} \sqrt{1+w^{-2}}-m^{j} \sqrt{1+m^{-2}} \begin{cases}\geq 0, & \text { if } j=0 \\ \leq 0, & \text { if } j \geq 1\end{cases}
$$

Using similar arguments to those in the proof of Lemma 3.5,

$$
F=\sum_{j=0}^{k} \rho_{j}\left(w^{j} \sqrt{1+w^{-2}}-m^{j} \sqrt{1+m^{-2}}\right)
$$




$$
\begin{aligned}
& \geq\left(\sqrt{1+w^{-2}}-\sqrt{1+m^{-2}}\right) \\
&+\rho\left(\sqrt{1+w^{-2}} \sum_{j=1}^{k} w^{j}-\sqrt{1+m^{-2}} \sum_{j=1}^{k} m^{j}\right) \\
&=\left(\sqrt{1+w^{-2}}-\sqrt{1+m^{-2}}\right) \\
&+\rho\left(\frac{\sqrt{1+w^{-2}}\left(w-w^{k+1}\right)}{1-w}-\frac{\sqrt{1+m^{-2}}\left(m-m^{k+1}\right)}{1-m}\right) \\
&=\left(\sqrt{1+w^{-2}}-\sqrt{1+m^{-2}}\right)+\rho\left(\frac{w \sqrt{1+w^{-2}}}{1-w}-\frac{m \sqrt{1+m^{-2}}}{1-m}\right) \\
&\left.\quad+\frac{m^{k+1} \sqrt{1+m^{-2}}}{1-m}-\frac{w^{k+1} \sqrt{1+w^{-2}}}{1-w}\right) \\
& \geq\left(\sqrt{1+w^{-2}}-\sqrt{1+m^{-2}}\right)+\rho\left(\frac{w \sqrt{1+w^{-2}}}{1-w}-\frac{m \sqrt{1+m^{-2}}}{1-m}\right) \\
& \geq 0
\end{aligned}
$$

if

$$
\rho \leq \frac{\left(\sqrt{1+w^{-2}}-\sqrt{1+m^{-2}}\right)(1-w)(1-m)}{m(1-w) \sqrt{1+m^{-2}}-w(1-m) \sqrt{1+w^{-2}}} \stackrel{\text { def }}{=} \tilde{F}(w) .
$$

Because $\tilde{F}(w)$ is decreasing in $w$,

$$
\min \tilde{F}(w)=\lim _{w \rightarrow m} \tilde{F}(w)=\frac{(1-m)^{2}}{m^{2}+m^{3}} .
$$

Hence, $F \geq 0$ when the condition (9) is satisfied.

COROLLARY 3.8. Let $T$ be a ramp network with a given topology. If the cost function $C(T)$ satisfies condition (9), then it has a unique local minimum.

\section{Discussion}

(1) The two fundamental properties (P1) and (P2) are important because the first property makes the design of optimal ramps simple given the positions of the Steiner points and the second property ensures that any known descent algorithms for locally minimizing the cost function for a given topology can be applied to the design of optimal ramp networks without the possibility of finding suboptimal 
local minimima.

(2) The techniques used in the previous section merit a brief discussion. The characteristics of the problem allow the interval for the values of the parameter $w_{i}$, the endpoint-gradient of $e_{i}$, to be divided into two parts that convert the cost function $C(T)$ into the form

$$
C(T)=\sum_{i} c^{*} \max \left\{F_{1 i}, F_{2 i}\right\}
$$

under condition (9) (Lemma 3.7). This expression for $C(T)$ effectively removes the gradient constraint on edges. Hence the problem of finding a minimum cost ramp network now becomes an unconstrained optimisation problem. Moreover, it follows from this expression that in order to prove the convexity of $C(T)$ it suffices to prove the convexity of $F_{1 i}, F_{2 i}$ separately.

(3) Condition (9) is a very weak condition which is easily satisfied in all known applications. If $m \leq 1 / 7$, Condition (9) becomes

$$
\max _{j}\left\{\rho_{i j}\right\} \leq \frac{(1-m)^{2}}{m^{2}+m^{3}} \leq \frac{(1-1 / 7)^{2}}{m\left(1 / 7+(1 / 7)^{2}\right)}=\frac{4.5}{m} .
$$

In all applications known to the authors, the constants $\rho_{i j}$ are much less than this bound. For example, the haulage cost provided by one Australian mining contractor is $(0.5+0.3 \mathrm{w} / \mathrm{m}) /(k T \cdot M)$, i.e. $c_{0}=$ $0.5, c_{1}=0.3$ and $c_{j}=0$ for $j>1$. Hence,

$$
\rho_{i 1}=\frac{c_{h} t\left(e_{i}\right) c_{1}}{c_{d}+c_{h} t\left(e_{i}\right) c_{0}} \leq \frac{c_{1}}{c_{0}}=\frac{0.6}{m}, \rho_{i j}=0(j>1) .
$$

We can see that Condition (17) is easily satisfied and hence, for this haulage cost, $C(T)$ is convex.

$R E M A R K$ 4.1. It is easily seen that in the linear case $(k=1)$ Inequality (14) becomes

$$
\frac{d^{2}}{d t^{2}} F_{1}(t)=\frac{\beta^{2}\left(1+w^{2}\right)}{L^{3}}\left(1-\rho w^{3}\right) \geq 0,
$$

and the condition for $\rho$ becomes $\rho \leq 1 / w^{3}$. The convexity of the cost function in this linear case has been discussed in (Brazil et al., 2000-2). 


\section{A Generalised Model: Underground Mine Networks with Ramps and Vertical Shafts}

The model of networks studied above can be generalised to those underground mine networks that consist not only of ramps but also contain vertical shafts. Figure 2 gives a practical example in Australia. The paper (Brazil et al., 2002) discusses the ramp network model with a linear haulage cost function, so here we give a brief description of the generalisation of the model discussed in this paper in which the haulage cost function is polynomial.

In the generalised model the points on the shafts where ore is loaded from the ramps are referred to as shaft access points, which become new variables in the generalised model. (More precisely, for a shaft access point, the variable is the depth of the shaft to that point, i.e. the distance between the shaft access point and the surface.) Let $T=T_{s} \cup T_{r}$ be a mining network in the generalised model where:

- $T_{s}$ is the subnetwork consisting of all shafts, and

- $T_{r}$ is the subnetwork consisting of the ramps.

The parameters in $T_{s}$ are shaft access points. Both the development costs and the haulage costs associated with a shaft are linear in the depth of the shaft. Therefore, the cost $C\left(T_{s}\right)$ is a convex function with respect its variables - the shaft-section lengths.

Notice that for any edge $(i, j)$ in $T_{r}$ the configuration space of its pair of endpoints $v(i), v(j)$ is a Cartesian product of two copies of $\mathbf{R}^{3}$. Keeping one endpoint fixed at the origin corresponds to projecting onto $\mathbf{R}^{3}$ by mapping $(v(i), v(j))$ to $v(i)-v(j)$. Let $\widetilde{C}(T)$ denote the induced cost function on $\mathbf{R}^{3}$ under this map. Now it is easy to see that $C(T)$ is convex on $\mathbf{R}^{3} \times \mathbf{R}^{3}$ if and only if $\widetilde{C}(T)$ is convex on $\mathbf{R}^{3}$. The same method works if we have one endpoint, say $v(j)$, representing a shaft access point. For in this case, $v(j)$ is free to move along a vertical line, i.e., a copy of $\mathbf{R}^{1}$. So the configuration of pairs of endpoints is $\mathbf{R}^{3} \times \mathbf{R}^{1}$ and the same projection method works, allowing us to keep the end at the shaft fixed. This relies on the fact that the endpoint $v(j)$ is restricted to a linear subspace of $\mathbf{R}^{3}$.

Hence it follows that we can use the arguments in Section 4 to show that $C\left(T_{r}\right)$ is a convex function under the condition (9). Hence, we conclude that in the generalised model the total cost $C(T)=C\left(T_{s}\right)+C\left(T_{r}\right)$ is a convex function under (9), and as indicated in Section 4, this property holds in real mining applications. 


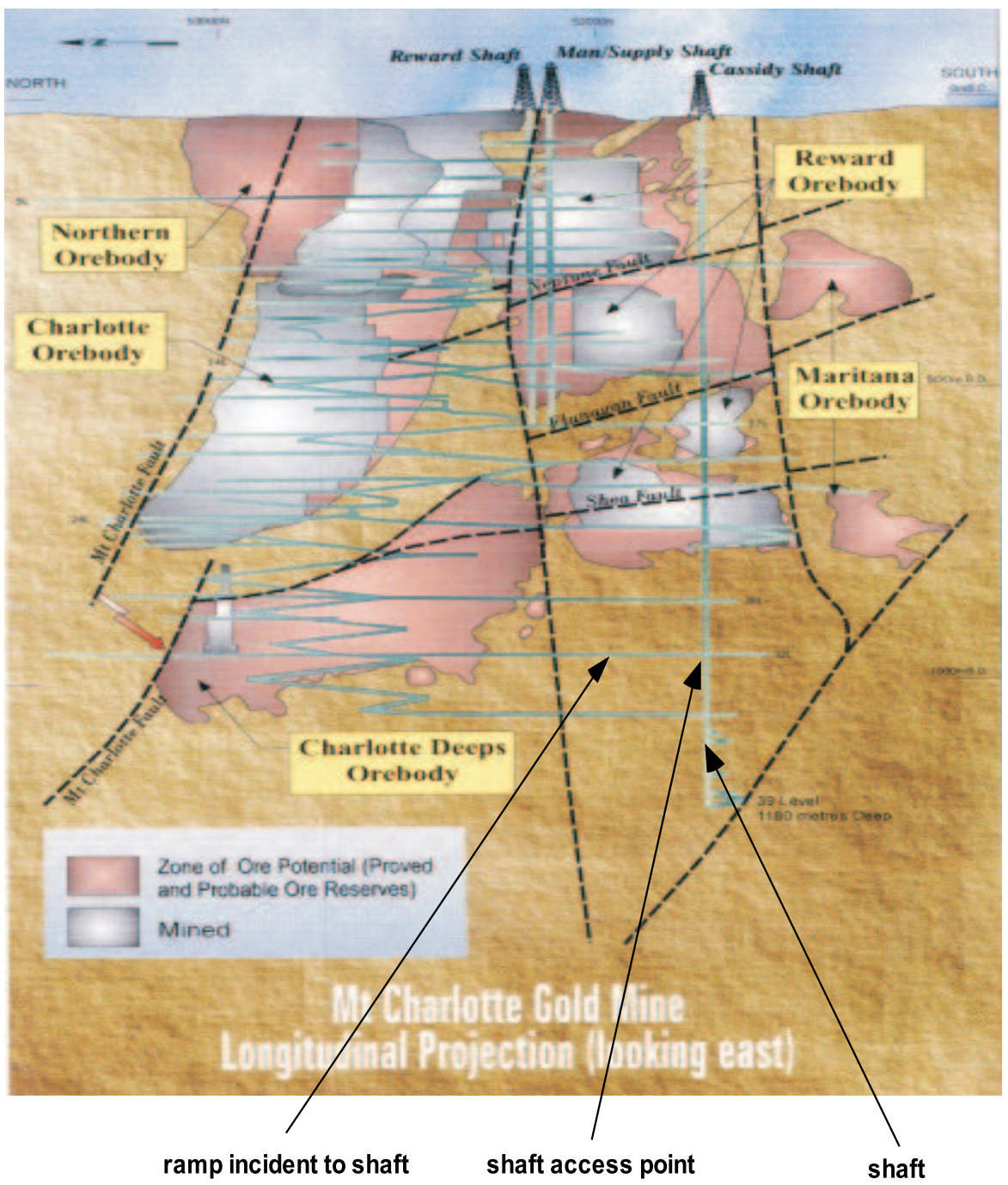

Figure 2. An underground network consisting of ramps and shafts.

\section{Acknowledgement}

The authors wish to thank N.C. Wormald for helpful discussions on this paper.

\section{References}

Brazil, M., D. A. Thomas, and J. F. Weng. Gradient constrained minimal Steiner trees. Network Design: Connectivity and Facilities Location (DIMACS Series in Discrete Mathematics and Theoretical Computer Science, American Mathematical Society, 40: 23-38, 1998. 
Brazil, M., J. H. Rubinstein, D. A. Thomas, J. F. Weng, and N. C. Wormald. Gradient-constrained minimal Steiner trees (I). Fundamentals. J. of Global Optimization, 21: 139-155, 2000.

Brazil, M., D. Lee, J. H. Rubinstein, D. A. Thomas, J. F. Weng, and N. C. Wormald. Network optimisation of underground mine design. The Australasian Institute for Mining and Metallurgy Proc., 305(1): 57-65, 2000.

Brazil, M., D. Lee, J. H. Rubinstein, D. A. Thomas, J. F. Weng, and N. C. Wormald. A network model to optimise cost in underground mine design. Trans. of the South African Institute of Electrical Engineering, 93(2): 97-103, 2002.

Clegg, J. C. Calculus of variation. Oliver and Boyd Ltd., Edinburgh, 1968.

Hwang, F. K., D. S. Richards, and P. Winter The Steiner tree problem, Annals of Discrete Mathematics 53. Elsevier, Amsterdam, 1992.

Lee, D. H. Industrial case studies of Steiner trees. paper presented at NATO Advanced Research Workshop on Topological Network Design, Denmark, 1989.

Normandy Mining Ltd. Q1 report on activities to shareholders (three months to 30 September, p. 5, 2001. 